\title{
The Bernstein-Sato $b$-function of the Space of Cyclic Pairs
}

\author{
by
}

\author{
Robin WALTERS
}

\begin{abstract}
We compute the Bernstein-Sato polynomial of $f$, a function which given a pair $(M, v)$ in $X=M_{n}(\mathbb{C}) \times \mathbb{C}^{n}$ tests whether $v$ is a cyclic vector for $M$. The proof includes a description of shift operators corresponding to the Calogero-Moser operator $L_{k}$ in the rational case.
\end{abstract}

2010 Mathematics Subject Classification: Primary 14F10; Secondary 32S25.

Keywords: Bernstein-Sato polynomial, $b$-function, cyclic pair, Calogero-Moser.

\section{$\S 1$. Introduction}

Let $f$ be an algebraic function on a variety $X$ over $\mathbb{C}$. Let $D_{X}$ be the ring of algebraic differential operators on $X$. The Bernstein-Sato b-function of $f$ is defined to be the minimal degree monic function $b(s)$ in $\mathbb{C}[s]$ such that

$$
D f^{s+1}=b(s) f^{s}
$$

for some operator $D$ in $D_{X}[s]=\mathbb{C}[s] \otimes D_{X}$. We call $D$ the Bernstein operator and (1.1) the Bernstein equation. A minimal $b(s)$ must exist since the set of all $b(s)$ satisfying (1.1) form an ideal in $\mathbb{C}[s]$. Existence of non-zero solutions to (1.1) was proved by Bernstein in 1971 [1]. The rationality of the roots of $b(s)$ was proved by Kashiwara in 1976 [9]. The $b$-function is interesting, in part, because it is an invariant of the singularities of the divisor given by $f$.

In [11], Opdam proves a conjecture of Yano and Sekiguchi [14] by computing the $b$-function corresponding to $I=\prod_{\alpha \in R^{+}} \alpha^{2}$, a $W$-invariant function on $\mathfrak{h}$. (The notation is defined in Section 5.) In type $A_{n}$, the function $I$ is the square of the

Communicated by T. Mochizuki. Received April 17, 2014. Revised November 5, 2014.

R. Walters: Department of Mathematics, University of Chicago,

5734 S. University Avenue, Chicago, IL 60637, USA;

e-mail: robin@math.uchicago.edu

(C) 2015 Research Institute for Mathematical Sciences, Kyoto University. All rights reserved. 
Vandermonde determinant. Opdam proves the result by realizing the Bernstein operator as a shift operator related to the Calogero-Moser operator.

For this paper, let $X=M_{n}(\mathbb{C}) \times \mathbb{C}^{n}$. For $(M, v) \in X$, we say $v$ is cyclic for $M$ or that $(M, v)$ is a cyclic pair if the set $\left\{v, M v, M^{2} v, \ldots\right\}$ is a spanning set of $\mathbb{C}^{n}$. Let $C(M, v)$ denote the square matrix

$$
\left[\begin{array}{llll}
v & M v M^{2} v & \ldots & \left.M^{n-1} v\right] .
\end{array}\right.
$$

We define $f(M, v)=\operatorname{det}(C(M, v))$, a polynomial on $X$. Then $(M, v)$ is cyclic if and only if $f(M, v) \neq 0$.

The function $f$ maps to $I$ via radial reduction. Since radial reduction maps the standard Laplacian operator $\Delta$ to the Calogero-Moser operator on $\mathfrak{h}[5]$, we can think of $X$ and $f$ as describing a broader, yet simpler, precursor situation to the one studied in [11]. More generally, the function $f$ and the space $X$ are relevant to the study of mirabolic $D$-modules and rational Cherednik algebras [2-4].

The main result of the paper is the computation of the $b$-function of $f$.

Theorem 1.1. The b-function of $f$ is

$$
\tilde{b}(s)=\prod_{0 \leq c<d \leq n}(s+1+c / d) .
$$

Since $f$ is a semi-invariant, the calculation has some similarity to the prehomogeneous case originally considered by Sato in [12] and [13]. In that case, $G$ acts on a vector space with open dense orbit. However, our space is not prehomogeneous, so some additional work is required.

The proof will proceed in three parts. First we will define a differential operator $S$ in $D_{X}[s]$, and show that one has an equation

$$
S f^{s+1}=b^{\prime}(s) f^{s}
$$

for an unknown function $b^{\prime}(s)$ in $\mathbb{C}[s]$. Secondly, we show $\tilde{b}(s)$, our specific candidate function, is the monic associate of $b^{\prime}(s)$. Thirdly, we will show that $\tilde{b}(s)$ has, in fact, the minimal degree, completing the proof.

Section 2 contains some results about $f$ and cyclic vectors. Then we continue to the proof of our main theorem. This is the content of Sections 3 through 6. In the Appendix (Section 7), we will give a proof characterizing the structure of the space of shift operators of the rational Calogero-Moser operator, an analog of a similar result for the trigonometric case in [11].

\section{$\S 2$. Cyclic vectors and semi-invariants}

The following result is well-known. 
Proposition 2.1. The matrix $M$ has a cyclic vector if and only if each Jordan block $B_{i}$ has a distinct eigenvector $\lambda_{i}$, that is, if each eigenspace is onedimensional.

We conjecture that $f$ is irreducible. The above result allows us to prove something weaker but still sufficient for our purposes. Let $\mathfrak{g}=M_{n}(\mathbb{C})$.

Proposition 2.2. The function $f$ has no non-constant, proper factor $h \in \mathbb{C}[\mathfrak{g}]$.

Proof. Such a proper factor would correspond to a set of codimension 1 in $\mathfrak{g}$ containing matrices $M$ with no cyclic vector. By the assumption that at least two Jordan blocks share an eigenvalue, we have $k-1$ choices of $\lambda_{i}$. Assuming $\lambda_{1}=\lambda_{2}=\lambda$ and reordering the basis so that the true eigenvectors of $B_{1}$ and $B_{2}$ are the first two vectors followed by all the generalized eigenvectors, we see that $B_{1} \oplus B_{2}$ is

$$
\left(\begin{array}{c|c}
\lambda \mathrm{Id}_{2} & A \\
\hline 0 & B
\end{array}\right) .
$$

The submatrix $B$ is upper triangular with $\lambda$ on its diagonal, and the entries of $A$ and of $B$ above the diagonal are 1 or 0 . Then this matrix is stabilized by

$$
\left(\begin{array}{c|c}
\mathrm{GL}(2) & 0 \\
\hline 0 & \mathbb{C}^{*} \mathrm{Id}_{n-2}
\end{array}\right) .
$$

Thus the dimension of this partition is less than or equal to

$$
n^{2}+(k-1)-(k-2)-1-4=n^{4}-4 .
$$

So we are done.

Given a space $Y$ with a $G$-action, we denote the $G$-invariant operators on $Y$ as $D_{Y}^{G}$. Denote $p^{g}(y)=p(g \cdot y)$. The function $p$ is said to be a $G$-semi-invariant corresponding to a character $\chi$ if

$$
p^{g}=\chi(g) p \quad \text { for all } g \in G .
$$

We denote the semi-invariant functions corresponding to $\chi$ as $\mathbb{C}[Y]^{\chi}$ and differential operators corresponding to $\chi$ as $D_{Y}^{\chi}$.

In our case, we have $G=\mathrm{GL}_{n}(\mathbb{C})$ acting on $X=\mathfrak{g l}_{n}(\mathbb{C}) \times \mathbb{C}^{n}$ via conjugation on the first factor and multiplication on the second factor. The one-dimensional representations (or characters) are just powers of the determinant, $\chi=\operatorname{det}^{r}$. Note that $f \in \mathbb{C}[X]^{\text {det }}$. The space of diagonal matrices in $\mathfrak{g l}_{n}(\mathbb{C})$ is isomorphic to $\mathbb{C}^{n}$. We denote it by $\mathfrak{h}$. Let $Z=\mathfrak{h} \times V \subset X$.

The following result is originally due to Weyl, although we give a different proof. 
Proposition 2.3. Let $p \in \mathbb{C}(s)[X]^{\chi}$ where $\chi=\operatorname{det}^{r}$. Then

$$
p=f^{r} h
$$

where $h \in \mathbb{C}(s)[\mathfrak{g}]^{G}$.

Proof. Consider $q=p f^{-r} \in \mathbb{C}(s)(X)^{G}$.

Now define the matrix

$$
d=\operatorname{diag}(1, \ldots, 1, \lambda, 1, \ldots, 1) \in G
$$

containing $\lambda$ in its $i^{\text {th }}$ entry. This acts on $Z$ by fixing $\mathfrak{h}$ and scaling $v^{i}$, the $i^{\text {th }}$ coordinate of $V$. However, since $\left.q\right|_{Z}$ is invariant, it must be homogeneous of degree 0 in $v^{i}$, that is, it is independent of $v^{i}$. By invariance, $q$ is independent of $v^{i}$ on $G \cdot Z$, which is dense in $X$, and thus $q \in \mathbb{C}(s)(\mathfrak{g})^{G}$.

It is not hard to see that

$$
\mathbb{C}(s)(\mathfrak{g})^{G}=\left\{\frac{a}{b} \mid a, b \in \mathbb{C}(s)[\mathfrak{g}]^{G}\right\}
$$

since a general element of $\mathbb{C}(s)(\mathfrak{g})^{G}$ is a ratio of semi-invariants, but $\mathbb{C}(s)[\mathfrak{g}]$ has no non-invariant semi-invariants.

So we know

$$
p=f^{r} \frac{a}{b}
$$

where $a, b \in \mathbb{C}(s)[\mathfrak{g}]^{G}$ and are, we can assume, relatively prime. This equation implies that $b$ divides $f^{r}$. However, by Proposition $2.2, f^{r}$ has no factor in $\mathbb{C}[\mathfrak{g}]$ and thus none in $\mathbb{C}(s)[\mathfrak{g}]$ either.

\section{§3. The operator $S$}

When we use the term order in reference to a differential operator in $D_{X}[s]$, we refer to the traditional filtration in which $\partial_{x_{i}}=\partial / \partial x_{i}$ has degree 1 and functions in $\mathbb{C}[s][X]$ have degree 0 . We will also refer to a $\mathbb{Z}$-grading on $D_{X}[s]$ in which $\left|\partial_{x_{i}}\right|=-1$ and $\left|x_{i}\right|=1$. The ring $\mathbb{C}[s]$ lives in the grade 0 . Note that the grading is well-defined on $D_{X}[s]$ since it respects the defining relation $\partial_{x_{i}} x_{i}-x_{i} \partial_{x_{i}}=1$. When we refer to this grading, we will use term degree.

We define a differential operator $S$ which is given by taking the function $f$ and replacing all variables with the corresponding partial derivatives. That is,

$$
S=\operatorname{det}\left(\left[\begin{array}{llllll}
\partial_{v} & \partial_{M} \partial_{v} & \partial_{M}^{2} \partial_{v} & \ldots & \partial_{M}^{n-1} \partial_{v}
\end{array}\right]\right)
$$

where $\left[\partial_{v}\right]_{i}=\partial_{v_{i}}$ and $\left[\partial_{M}\right]_{j}^{i}=\partial_{m_{j}^{i}}$. Then $S$ is an order $n(n+1) / 2$ differential 
operator and $S \in D_{X}^{\text {det }^{-1}}$. The definition of $S$ here is analogous to that of the Bernstein operator in Sato's prehomogeneous case.

We can now prove the following:

Proposition 3.1. There exists a function $b^{\prime} \in \mathbb{C}[s]$ such that $S f^{n+1}=b^{\prime}(s) f^{n}$. Further, $\operatorname{deg}\left(b^{\prime}(s)\right) \leq n(n+1) / 2$.

Proof. Since $S$ has weight $\operatorname{det}^{-1}$ and $f^{s+1}$ has weight $\operatorname{det}^{s+1}$, the operator $S \circ \hat{f}^{s+1}$ has weight $\operatorname{det}^{s}$. Applying this operator to 1 , we get a polynomial $p \in \mathbb{C}[s][X]^{\text {det }^{s}}$. Therefore by Proposition 2.3 , there exists $q \in \mathbb{C}[s][X]^{G}$ such that

$$
S f^{s+1}=f^{s} q
$$

Taking the degree of both sides we see

$$
-\frac{n(n+1)}{2}+(s+1) \frac{n(n+1)}{2}=s \frac{n(n+1)}{2}+\operatorname{deg}(q) .
$$

Thus $\operatorname{deg}(q)=0$ and so $q \in \mathbb{C}[s]$ as desired. Denote $q$ as $b^{\prime}(s)$. Moreover since $S$ is an $n(n+1) / 2$-order operator, we see $\operatorname{deg}\left(b^{\prime}\right) \leq n(n+1) / 2$.

\section{§4. Localization}

In this and the next section, we will show that $b^{\prime}(s)$ equals

$$
\tilde{b}(s) \alpha_{n}=\prod_{0 \leq c<d \leq n}(d(s+1)+c),
$$

where $\alpha_{n}=\prod_{d=1}^{n} d^{d} \in \mathbb{C}$. We factor this as:

$$
\begin{aligned}
& b_{1}(s)=n !(s+1)^{n}, \\
& b_{2}(s)=\prod_{1 \leq c<d \leq n}(d(s+1)+c),
\end{aligned}
$$

so that $b_{1} b_{2}=\tilde{b} \alpha_{n}$.

Proposition 4.1. The polynomial $b_{1}(s)$ divides the b-function of $f$.

Proof. Consider the following local coordinates for $X$ :

$$
\begin{gathered}
\mathbb{C}^{n(n-1)} \times \mathbb{C}^{n} \times \mathbb{C}^{n} \rightarrow X, \\
\varphi:\left(\left\{t_{j}^{i}\right\}_{i \neq j},\left\{a_{i}\right\}_{i=1}^{n},\left\{v_{i}\right\}_{i=1}^{n}\right) \mapsto\left(T A T^{-1}, T v\right),
\end{gathered}
$$


where

$$
T=\left(\begin{array}{ccccc}
1 & t_{2}^{1} & t_{3}^{1} & \ldots & t_{n}^{1} \\
t_{1}^{2} & 1 & t_{3}^{2} & \ldots & t_{n}^{2} \\
t_{1}^{3} & t_{2}^{3} & 1 & \ldots & t_{n}^{3} \\
\vdots & \vdots & \vdots & \ddots & \vdots \\
t_{1}^{n} & t_{2}^{n} & t_{3}^{n} & \ldots & 1
\end{array}\right) \quad \text { and } \quad A=\left(\begin{array}{ccccc}
a_{1} & 0 & 0 & \ldots & 0 \\
0 & a_{2} & 0 & \ldots & 0 \\
0 & 0 & a_{3} & \ldots & 0 \\
\vdots & \vdots & \vdots & \ddots & \vdots \\
0 & 0 & 0 & \ldots & a_{n}
\end{array}\right)
$$

Let $p \in X$ be defined by $t_{j}^{i}=0, a_{i}=i$ and $v_{i}=0$. It is straightforward to compute that $\left.\operatorname{det}(D \varphi)\right|_{p} \neq 0$, and thus $\varphi$ gives local coordinates at $p$.

In these local coordinates we have

$$
f=\operatorname{det}(T) \operatorname{det}(C(A, v)) .
$$

Note $C(A, v)_{j}^{i}=v_{i} a_{i}^{j-1}$ is the Vandermonde matrix with rows multiplied by $v_{i}$. So

$$
\operatorname{det}(C(A, v))=v_{1} \cdots v_{n} \prod_{1 \leq i<j \leq n}\left(a_{j}-a_{i}\right)
$$

Let $\overline{1}=(1, \ldots, 1)$. Since at $p, a_{i}=i$, the quantity

$$
\operatorname{det}(C(A, \overline{1}))=\prod_{1 \leq i<j \leq n}\left(a_{j}-a_{i}\right) \neq 0 .
$$

Similarly, $T=I_{n}$ at $p$, so $\operatorname{det}(T)=1$. Thus in a small open neighborhood $U$ of $p$, the function $\operatorname{det}(T) \operatorname{det}(C(A, \overline{1}))$ is invertible.

We will show that the $b$-function for $f$ on $U$ is $b_{1}(s)$, which will finish the proof since the global $b$-function is the least common multiple of the local $b$-functions [6, Proposition 2.1].

Let

$$
D=\operatorname{det}(C(A, \overline{1}))^{-1} \operatorname{det}(T)^{-1} \partial_{v_{1}} \ldots \partial_{v_{n}}
$$

Then

$$
D f^{s+1}=b_{1}(s) f^{s} \text {. }
$$

Since $v_{i}$ are not invertible near $p$, the Bernstein operator $D$ must include the factor $\partial_{v_{1}} \ldots \partial_{v_{n}}$. Thus $b_{1}$ is minimal.

\section{$\S 5$. Radial parts reduction}

For $b_{2}$, we relate our problem to the situation of [11]. To do this we require the radial parts map from [5, Appendix].

We introduce more notation. Denote by $R$ and $R^{+}$the sets of roots and of positive roots respectively for type $A_{n-1}$. Let $W=S_{n}$ be the Weyl group 
corresponding to $\mathfrak{g l}_{n}$. Consistent with the notation in [5], we define $X^{\text {reg }}$ to consist of pairs $(M, v)$ where $M \in \mathfrak{g}^{\text {rs }}$. Lastly define $\mathfrak{h}^{\text {reg }} \subset \mathfrak{h}$ to be those points which avoid the root hyperplanes.

The radial parts map is more clearly described in [5]. We give an overview. To derive it, we start with the map

$$
\rho: X^{\text {reg }} / G \rightarrow \mathfrak{h}^{\text {reg }} / W, \quad(M, v) \mapsto \text { eigenvalues of } M,
$$

which induces the map

$$
\rho^{*}: \mathbb{C}\left[\mathfrak{h}^{\mathrm{reg}}\right]^{W} \rightarrow \mathbb{C}\left[X^{\mathrm{reg}}\right]^{G} .
$$

For $k \in \mathbb{C}$, we define the radial parts map $\operatorname{Rad}_{k}: D_{X}^{G} \rightarrow D_{\mathfrak{h}^{\text {reg }}}^{W}$ as follows. Let $D \in D_{X}^{G}$ and $g \in \mathbb{C}\left[\mathfrak{h}^{\text {reg }}\right]^{W}$. Then

$$
\operatorname{Rad}_{k}(D)(g)=\left.f^{-k} D\left(f^{k} \rho^{*}(g)\right)\right|_{\mathfrak{h}} .
$$

Let $\delta=\left.f\right|_{\mathfrak{h}^{\text {reg }}}$ be the Vandermonde determinant and $L_{k}$ be the Calogero-Moser operator

$$
L_{k}=\Delta_{\mathfrak{h}}-\sum_{\alpha \in R^{+}} k(k+1) \frac{(\alpha, \alpha)}{\alpha^{2}} .
$$

Note that since $\delta$ is the product of the positive roots, $\alpha^{-1}$ as well as $\delta^{-1}$ are in $\mathbb{C}\left[\mathfrak{h}^{\text {reg }}\right]$. Let $\Delta_{\mathfrak{g}}$ be the standard Laplacian on $\mathfrak{g}$. Since $X=\mathfrak{g} \times V$, we can view $\Delta_{\mathfrak{g}}$ as a differential operator on $X$. By [5, Appendix],

$$
\operatorname{Rad}_{k}\left(\Delta_{\mathfrak{g}}\right)=\delta^{-k-1} L_{k} \delta^{k+1} .
$$

Define $P^{+}=\sum_{\alpha \in R^{+}} \alpha^{-1} \partial_{X_{\alpha}}$. Then we can simplify (5.1) using the equations $\delta^{-1} \Delta_{\mathfrak{h}} \delta=\Delta_{\mathfrak{h}}+2 P^{+}$and $\delta^{-1} P^{+} \delta=P^{+}+2 \sum_{\alpha \in R^{+}}(\alpha, \alpha) \alpha^{-2}$ to get

$$
\operatorname{Rad}_{k}\left(\Delta_{\mathfrak{g}}\right)=\Delta_{\mathfrak{h}}+2(k+1) P^{+},
$$

an operator which we will call $L_{\mathfrak{h}}(k+1)$.

We say that $D \in D_{\mathfrak{h}^{\text {reg }}}^{W} \otimes \mathbb{C}[k]$ is a shift operator if

$$
D L_{\mathfrak{h}}(k)=L_{\mathfrak{h}}(k+r) D .
$$

Denote the set of shift operators with shift $r$ as $\mathbb{S}_{\mathfrak{h}}(r, k)$, or just $\mathbb{S}_{\mathfrak{h}}(r)$ if $k$ is clear from context. Note that $\mathbb{S}_{\mathfrak{h}}(0)$ is just the centralizer of $L_{\mathfrak{h}}(k)$.

The following is a rational case analog to the trigonometric case given in [11, Theorem 3.1].

Proposition 5.1. The set $\mathbb{S}_{\mathfrak{h}}(r, k)$ is a free, rank-one $\mathbb{S}_{\mathfrak{h}}(0, k)$-module.

We defer the proof until Section 7. We denote the single generator of $\mathbb{S}_{\mathfrak{h}}(r, k)$ over $\mathbb{S}_{\mathfrak{h}}(0, k)$ by $g(r, k)$. We denote the operator $p \mapsto f \cdot p$ by $\hat{f}$. 
Proposition 5.2. The operator $\operatorname{Rad}_{k}(S \hat{f})$ belongs to $\mathbb{S}_{\mathfrak{h}}(-1, k+2)$.

Proof. As noted before, $S \hat{f}$ is a semi-invariant with character $\operatorname{det} \cdot \operatorname{det}^{-1}=1$, so $S \hat{f} \in D_{X}^{G}$, and thus we can take its radial part $\operatorname{Rad}(S \hat{f}) \in D_{\mathfrak{h}^{\text {reg }}}^{W}$.

The set $\mathbb{S}_{\mathfrak{h}}(-1, k+2)$ is a subset of $D_{\mathfrak{h}^{\text {reg }}}^{W} \otimes \mathbb{C}[k]$. So we need to show that $k \mapsto \operatorname{Rad}_{k}(S \hat{f})$ is a polynomial map $\mathbb{C} \rightarrow D_{\mathfrak{h}^{\text {reg }}}^{W}$. Given $p \in \mathbb{C}[X]$, we denote the corresponding differential operator as $\partial_{p}$ or $\partial(p)$. Let $p=x_{1} \cdots x_{m}$. Denote $(k+1)_{r}=(k+1) \ldots(k-r+2)$, the falling Pochhammer symbol. Then from the formula

$$
f^{-k} \partial_{p} f^{k+1} g=\sum_{q \cdot p_{1} \cdots p_{r}=p}(k+1)_{r} f^{1-r}\left(\prod_{i=1}^{r} \partial_{p_{i}} f\right) \partial_{q} g,
$$

we can see $\operatorname{Rad}_{k}(S \hat{f})$ is a polynomial in $k$, since $k$ appears only in the polynomial coefficients $(k+1)_{r}$.

Now we need to show that $\operatorname{Rad}_{k}(S \hat{f})$ satisfies (5.3). Since $S$ and $\Delta_{\mathfrak{g}}$ are constant coefficient operators, they commute, which gives the equation

$$
S \hat{f} \widehat{f-1} \Delta_{\mathfrak{g}} \hat{f}=\Delta_{\mathfrak{g}} S \hat{f} .
$$

Applying $\operatorname{Rad}_{k}$, which is a homomorphism, we get

$$
\operatorname{Rad}_{k}(S \hat{f}) \operatorname{Rad}_{k}\left(\widehat{f^{-1}} \Delta_{\mathfrak{g}} \hat{f}\right)=\operatorname{Rad}_{k}\left(\Delta_{\mathfrak{g}}\right) \operatorname{Rad}_{k}(S \hat{f}) .
$$

Thus we obtain

$$
\operatorname{Rad}_{k}(S \hat{f}) L_{\mathfrak{h}}(k+2)=L_{\mathfrak{h}}(k+1) \operatorname{Rad}_{k}(S \hat{f}),
$$

as required.

The operator $\operatorname{Rad}_{k}(S \hat{f})$ helps us compute $b^{\prime}(k)$, since

$$
\operatorname{Rad}_{k}(S \hat{f})(1)=\left.\left(f^{-k} S f^{k+1}\right)\right|_{\mathfrak{h}}=b^{\prime}(k) .
$$

We now introduce the relation to the trigonometric case studied in [11]. Let $H$ be the complex torus with $\operatorname{Lie}(H)=\mathfrak{h}$. For $h \in H$, denote $h^{\alpha}=\exp (\alpha)(h)$. We then set

$$
H^{\text {reg }}=\left\{h \in H \mid h^{\alpha} \neq 0 \text { for all } \alpha \in R^{+}\right\} .
$$

Define the trigonometric Calogero-Moser operator with parameter $k \in \mathbb{C}$ on $H^{\text {reg }}$ :

$$
L_{H}(k)=\Delta_{\mathfrak{h}}-\sum_{\alpha \in R^{+}} k \frac{1+h^{\alpha}}{1-h^{\alpha}} \partial\left(X_{\alpha}\right)
$$

We also define

$$
\rho(k)=k \frac{1}{2} \sum_{\alpha \in R^{+}} \alpha .
$$


Then we define the related operator $\tilde{L}_{H}(k)=L_{H}(k)+(\rho(k), \rho(k))$. Let $\mathbb{S}_{H}(r, k)$ be the space of shift operators with respect to this trigonometric operator,

$$
\mathbb{S}_{H}(r, k)=\left\{D \in D_{H}^{W} \mid D \tilde{L}_{H}(k)=\tilde{L}_{H}(k+r) D\right\} .
$$

The relationship to the rational case comes from the map $\epsilon: D_{H} \rightarrow D_{\mathfrak{h}}$ which takes the lowest homogeneous part of the operator with respect to the grading defined by the degree of the differential operator. This map is more carefully defined in [11, Section 3]. The lowest homogeneous degree or lhd of $\tilde{L}_{H}(k)$ is -2 , and so

$$
\epsilon\left(\tilde{L}_{H}(k)\right)=\Delta_{\mathfrak{h}}+2 k \sum_{\alpha \in R^{+}} \frac{1}{\alpha} \partial_{X_{\alpha}},
$$

which is $L_{\mathfrak{h}}(k)$ from above. Note that $\epsilon$ satisfies

$$
\begin{aligned}
\epsilon\left(D_{1} D_{2}\right) & =\epsilon\left(D_{1}\right) \epsilon\left(D_{2}\right) & & \text { for all } D_{1}, D_{2}, \\
\epsilon\left(D_{1}+D_{2}\right) & =\epsilon\left(D_{1}\right)+\epsilon\left(D_{2}\right) & & \text { for all } D_{1}, D_{2} \text { with } \operatorname{lhd}\left(D_{1}\right)=\operatorname{lhd}\left(D_{2}\right) .
\end{aligned}
$$

Thus if $D \in \mathbb{S}_{H}(r, k)$, we get

$$
\epsilon(D) L_{\mathfrak{h}}(k)=L_{\mathfrak{h}}(k+r) \epsilon(D) .
$$

So $\epsilon(D) \in \mathbb{S}_{\mathfrak{h}}(r, k)$.

From [11, Theorem 3.1] we know that $\mathbb{S}_{H}(r, k)$ is a rank-one $\mathbb{S}_{H}(0, k)$-module, similar to the case of $\mathbb{S}_{\mathfrak{h}}$. We denote the generator by $G(r, k)$. Moreover, we prove the following proposition in Section 7.

Proposition 5.3. The map $\epsilon$ sends $G(-1, k)$ to $g(-1, k)$.

Since $\operatorname{Rad}_{k}(S \hat{f}) \in \mathbb{S}_{\mathfrak{h}}(-1, k+2)$, we know there exists some $D_{0} \in \mathbb{S}_{\mathfrak{h}}(0, k+2)$ such that $D_{0} \cdot \epsilon(G(-1, k+2))=\operatorname{Rad}_{k}(S \hat{f})$. By [11, Theorem 3.3], we know $G(-1, k+2)$ has lowest homogeneous degree 0 . Thus it can be written

$$
G(-1, k+2)=\sum_{i \in \mathcal{I}} p_{i} \partial\left(q_{i}\right)
$$

for some $p_{i}$ and $q_{i}$, homogeneous polynomials with $\operatorname{deg}\left(p_{i}\right) \geq \operatorname{deg}\left(q_{i}\right)$. Thus

$$
\epsilon(G(-1, k+2))=\sum_{i} p_{i} \partial\left(q_{i}\right)
$$

where the sum is over $\left\{i \in \mathcal{I} \mid \operatorname{deg}\left(p_{i}\right)=\operatorname{deg}\left(q_{i}\right)\right\}$. Given a differential operator $P$, we define the constant term of $P$, denoted $\mathrm{CT}(P)$, to be the scalar part of the operator, i.e., the summand with order zero and degree zero. If we write $P=$ $\sum_{i} p_{i} \partial\left(q_{i}\right)$, then $\operatorname{CT}(P)=p_{k} \partial\left(q_{k}\right)$ where $\operatorname{deg}\left(p_{k}\right)=\operatorname{deg}\left(q_{k}\right)=0$. We see that

$$
\epsilon(G(-1, k+2)) \cdot 1=\mathrm{CT}(\epsilon(G(-1, k+2)))=\mathrm{CT}(G(-1, k+2)) .
$$


Let $r_{0}=\mathrm{CT}\left(D_{0}\right) \in \mathbb{C}[k]$. Then

$$
\begin{aligned}
\mathrm{CT}\left(\operatorname{Rad}_{k}(S f)\right) & \left.=\operatorname{Rad}_{k}(S f) \cdot 1=D_{0} \epsilon(G(-1, k+2))\right) \cdot 1 \\
& =D_{0} \cdot \mathrm{CT}(G(-1, k+2))=r_{0} \mathrm{CT}(G(-1, k+2)) .
\end{aligned}
$$

We deduce that $\mathrm{CT}(G(-1, k+2))$ divides $\mathrm{CT}\left(\operatorname{Rad}_{k}(S f)\right)=b^{\prime}(k)$.

Let $\Gamma$ be the Gamma function. For a reduced root system define

$$
\tilde{c}(\lambda, k)=\prod_{\alpha \in R^{+}} \frac{\Gamma\left(-\left(\lambda, \alpha^{\vee}\right)\right)}{\Gamma\left(-\left(\lambda, \alpha^{\vee}\right)+k\right)} .
$$

In the special case of type $A_{n}$ we have

$$
\frac{1}{\tilde{c}(-\rho(k), k)}=\prod_{d=2}^{n} \frac{\Gamma(d k)}{\Gamma(k)} .
$$

By [11, Theorem 3.1, Corollary 3.4, Corollary 5.2], we know that

$$
\mathrm{CT}(G(-1, k+2))=\frac{\tilde{c}(-\rho(k+1), k+1)}{\tilde{c}(-\rho(k+2), k+2)} .
$$

So substituting (5.6) into (5.7) and canceling, we arrive at

$$
\mathrm{CT}(G(-1, k+2))=n ! \prod_{d=2}^{n} \prod_{j=1}^{d-1}(d(k+1)+j) .
$$

In summary, we have proved the following.

Proposition 5.4. The polynomial $b_{2}(s)=\prod_{d=2}^{n} \prod_{j=1}^{d-1}(d(s+1)+j)$ divides $b^{\prime}(s)$ in $\mathbb{C}[s]$.

Thus we have shown $b_{1}$ and $b_{2}$ divide $b^{\prime}$. Since $b_{1}$ and $b_{2}$ are coprime and $\operatorname{deg}(\tilde{b}) \leq n(n+1) / 2$, it follows that $b^{\prime}=\tilde{b} \alpha_{n}=b_{1} b_{2}$.

\section{$\S 6$. Minimality of $\tilde{b}$}

To complete the proof of Theorem 1.1, we must show that $\tilde{b}$ has minimal degree. In our case, this will follow directly from Proposition 6.1. Set $m=n(n+1) / 2=$ $\operatorname{deg}(f)$.

The proof that Sato's $b$-function in the prehomogeneous case gives the Bernstein polynomial (see, e.g., [8]) is general enough to cover our situation. The argument given in [8] proves the following result. 
Proposition 6.1. Let $f \in \mathbb{C}\left[x_{1}, \ldots, x_{n}\right]$ be homogeneous of degree $d$. If the operator $D \in \mathbb{C}\left[s, \partial_{x_{1}}, \ldots, \partial_{x_{n}}\right]$ satisfies (1.1) for some polynomial $b_{D}(s)$ and is not divisible by any non-scalar factor in $\mathbb{C}[s]$, then $b_{D}$ is the Bernstein polynomial for $f$.

Proof. The proof is identical to the proof of [8, Theorem 6.3.1]. We give a sketch of it for the reader's convenience.

For any polynomial $p(s)=\prod_{\mu}(s-\mu)$, we define

$$
\gamma_{p}(s)=\prod_{\mu} \Gamma(s-\mu) .
$$

Let $b(s)$ be a polynomial satisfying (1.1) with respect to $f \in \mathbb{C}[X]$. Define the zeta function corresponding to $f$ as

$$
Z_{f}(s)=\int_{X}|f(x)|^{s} e^{-2 \pi|x|^{2}} d x .
$$

By [8, Theorem 5.3.2], $Z_{f}(s) / \gamma_{b}(s)$ is holomorphic on $\mathbb{C}$. Each root of $b$ corresponds to infinitely many zeros of $Z_{f}(s) / \gamma_{b}(s)$. Thus if $Z_{f}(s) / \gamma_{b}(s)$ is nowhere-vanishing, $b$ must be minimal. We will now show this for $b_{D}$.

The integration by parts argument from [8, Theorem 6.3.1], applied with $D$ instead of $f(\partial)$, shows that

$$
Z_{f}(s)=(2 \pi)^{-d s} \gamma_{b_{D}}(s) \prod_{\mu} \frac{1}{\Gamma(-\mu)}
$$

for $\operatorname{Re}(s)>0$. Thus $Z_{f}(s) / \gamma_{b_{D}}(s)$ is a nowhere-vanishing holomorphic function.

Remark 1. Let $D \in \mathbb{C}\left[s, \partial_{x_{1}}, \ldots, \partial_{x_{n}}\right]$ be an operator satisfying (1.1) with respect to the Bernstein polynomial $b$. Let $\tilde{D}$ be the degree $-m$ part of $D$. Then taking the degree $m s$ part of (1.1) we get $\tilde{D} f^{s+1}=b(s) f^{s}$. Since $G$ acts locally finitely on $D_{X}$, we can write $\tilde{D}=\sum_{i} \tilde{D}_{i}$ where $g \cdot \tilde{D}_{i}=\operatorname{det}(g)^{i} \tilde{D}_{i}$. Then since $g f=\operatorname{det}(g) f$, this means $\tilde{D}_{i} f^{s+1}=0$ unless $i=-1$. Thus we have $\tilde{D}_{-1} f^{s+1}=b(s) f^{s}$. Then by Proposition 2.3, since $\operatorname{deg}(f)=\operatorname{deg}(S)$ we have

$$
\tilde{D}_{-1}=c S
$$

where $c \in \mathbb{C}$. So we have, in fact, shown that any Bernstein operator independent of $x \in X$ realizing $b(s)$ is our differential operator $S$ up to a constant factor.

\section{§7. Appendix: Proof of Propositions 5.1 and 5.3}

In this section, we will prove Propositions 5.1 and 5.3. 
Given $\mathbf{j} \in \mathbb{Z}^{R^{+}}$, an index on $R^{+}$, we define a partial order by $\mathbf{j} \leq 0$ if $\mathbf{j}_{\alpha} \leq 0$ for all $\alpha$. We represent the basis of $\mathbb{Z}^{R^{+}}$by $e_{\alpha}$ where $\alpha \in R^{+}$. Also denote $\bar{\alpha}^{\mathbf{j}}=\prod_{\alpha \in R^{+}} \alpha^{\mathbf{j}_{\alpha}}$. If $p \in \mathbb{C}[k] \otimes \mathbb{C}[\mathfrak{h}]$, then as above, we denote the corresponding differential operator in $\mathbb{C}[k] \otimes D_{\mathfrak{h}}$ as $\partial_{p}$ or $\partial(p)$.

Let $h_{1}, \ldots, h_{n}$ be an orthonormal basis of $\mathfrak{h}^{*}$. As a shorthand we write $\partial_{i}=$ $\partial\left(h_{i}\right)$. So $\Delta_{\mathfrak{h}}=\sum_{i=1}^{n} \partial_{i}^{2}$.

The following lemma is a straightforward calculation.

Lemma 7.1. Let $\sum \mathbf{r}=\sum_{i=1}^{n} \mathbf{r}_{i}$ and $p^{(\mathbf{r})}=\prod_{i=1}^{n} \partial_{t_{i}}^{\mathbf{r}_{i}} p(t)$ and

$$
\mathrm{AV}(\mathbf{r})=\frac{\left(\sum \mathbf{r}\right) !}{\prod_{i=1}^{n}\left(\mathbf{r}_{i} !\right)}(-1)^{\sum \mathbf{r}} .
$$

Then

$$
\partial(p)\left(\alpha^{-1} e^{t \lambda}\right)=\sum_{\mathbf{r}=0}^{\infty} \operatorname{AV}(\mathbf{r}) \alpha^{-1-\sum \mathbf{r}} \prod_{i=1}^{n}\left(\partial_{i} \alpha\right)^{r_{i}} p^{(\mathbf{r})}
$$

The key lemma we need for proving Proposition 5.1 reads as follows.

Lemma 7.2. The map

$$
p_{\mathbf{N}}: \mathbb{S}_{\mathfrak{h}}(-1, k) \rightarrow \mathbb{C}[k] \otimes \mathbb{C}[\mathfrak{h}], \quad \sum_{\mathbf{j} \leq \mathbf{N}} \bar{\alpha}^{\mathbf{j}} \partial\left(p_{\mathbf{j}}\right) \mapsto p_{\mathbf{N}},
$$

is injective.

Proof. First note that if $D \in \mathbb{S}_{\mathfrak{h}}(r, k)$ and $S \in \mathbb{S}_{\mathfrak{h}}(0, k)$, then commuting $S$ and $L_{\mathfrak{h}}(k)$ yields

$$
(D S) L_{\mathfrak{h}}(k)=L_{\mathfrak{h}}(k+r)(D S) .
$$

So $\mathbb{S}_{\mathfrak{h}}(r, k)$ is an $\mathbb{S}_{\mathfrak{h}}(0, k)$-module.

We will solve for an arbitrary element $D$ of the set $\mathbb{S}_{\mathfrak{h}}(r, k)$. Since $D$ is in $\mathbb{C}[k] \otimes D_{\mathfrak{h}^{\text {reg }}}^{W}$, we can write

$$
D=\sum_{\mathbf{j} \leq \mathbf{N}} \bar{\alpha}^{\mathbf{j}} \partial\left(p_{\mathbf{j}}\right) .
$$

Let $e^{t \lambda}=e^{t_{1} \lambda_{1}+\cdots+t_{n} \lambda_{n}}$. If $\alpha=\sum_{i} c_{i} \lambda_{i}$, then denote $t_{\alpha}=\sum_{i} c_{i} t_{i}$. Then applying (5.3) to $e^{t \lambda}$, we solve for $D$ (i.e. solve for the $p_{i}$ ) in

$$
D L_{\mathfrak{h}}(k) e^{t \lambda}=L_{\mathfrak{h}}(k+r) D e^{t \lambda} .
$$


Using Lemma 7.1, we expand this into

$$
\begin{aligned}
0=D L_{\mathfrak{h}}(k) e^{t \lambda}-L_{\mathfrak{h}}(k+r) D e^{t \lambda} & \\
=e^{t \lambda} \sum_{\mathbf{j} \leq \mathbf{N}} \bar{\alpha}^{\mathbf{j}}\left[2 k \sum_{\alpha \in R^{+}}\right. & t_{\alpha} \sum_{\mathbf{r}=0}^{\infty} \operatorname{AV}(\mathbf{r}) \alpha^{-1-\sum \mathbf{r}} \prod_{i}\left(\partial_{i} \bar{\alpha}\right)^{\mathbf{r}_{i}} p^{(\mathbf{r})}(t) \\
& -p_{\mathbf{j}}(t)\left\{\sum_{\alpha \in R^{+}}\left(\mathbf{j}_{\alpha}^{2}-\mathbf{j}_{\alpha}\right) \alpha^{-2}\left(\sum_{l=1}^{n}\left(\partial_{l} \alpha\right)^{2}\right)+2 \mathbf{j}_{\alpha} \alpha^{-1} t_{\alpha}\right. \\
& +2 \mathbf{j}_{\alpha} \sum_{\alpha \neq \beta} \mathbf{j}_{\beta} \alpha^{-1} \beta^{-1}\left(\sum_{l=1}^{n}\left(\partial_{l} \alpha\right)\left(\partial_{l} \beta\right)\right) \\
& \left.\left.+2(k+r)\left(\sum_{\alpha \in R^{+}} \alpha^{-2} \mathbf{j}_{\alpha} 2+\alpha^{-1} t_{\alpha}+2 \sum_{\alpha \neq \beta} \beta^{-1} \alpha^{-1} \mathbf{j}_{\alpha}\left(\partial_{\beta} \alpha\right)\right)\right\}\right] .
\end{aligned}
$$

Hence extracting the coefficient of $\bar{\alpha}^{\mathbf{N}-e_{\beta}} e^{t \lambda}$, we get

$$
0=p_{\mathbf{N}} t_{\beta} 2\left(k-\mathbf{N}_{\beta}-(k+r)\right)
$$

and so assuming that $p_{\mathbf{N}} \neq 0$ we find that $\mathbf{N}_{\beta}=-r$ for all $\beta$. That is, $\mathbf{N}=$ $(-r, \ldots,-r)$.

Denote $\nabla \alpha=\left(\partial_{1} \alpha, \ldots, \partial_{m} \alpha\right)$. In general, if we look at the coefficient of $\bar{\alpha}^{\mathbf{M}}$ we see that the following expression is 0 :

$$
\begin{aligned}
& 2 k \sum_{\alpha \in R^{+}} \sum_{\mathbf{r}=0}^{\infty} p_{\mathbf{M}+e_{\alpha}\left(1+\sum \mathbf{r}\right)}^{(\mathbf{r})} t_{\alpha} \operatorname{AV}(\mathbf{r}) \prod_{i=1}^{n}\left(\partial_{i} \alpha\right)^{\mathbf{r}_{i}} \\
& -\sum_{\alpha \in R^{+}} p_{\mathbf{M}+2 e_{\alpha}}\left(\mathbf{M}_{\alpha}+2\right)\left(\mathbf{M}_{\alpha}+1\right) \nabla \alpha \cdot \nabla \alpha-\sum_{\alpha \in R^{+}} p_{\mathbf{M}+e_{\alpha}} 2\left(\mathbf{M}_{\alpha}+1\right) t_{\alpha} \\
& -\sum_{\alpha \neq \beta} p_{\mathbf{M}+e_{\alpha}+e_{\beta}} 2\left(\mathbf{M}_{\alpha}+1\right)\left(\mathbf{M}_{\beta}+1\right) \nabla \alpha \cdot \nabla \beta-\sum_{\alpha \in R^{+}} p_{\mathbf{M}+2 e_{\alpha}} 4(k+r)\left(\mathbf{M}_{\alpha}+2\right) \\
& \quad-\sum_{\alpha \in R^{+}} p_{\mathbf{M}+e_{\alpha}} 2(k+r) t_{\alpha}-\sum_{\alpha \in R^{+}} p_{\mathbf{M}+e_{\alpha}+e_{\beta}} 4(k+r)\left(\mathbf{M}_{\alpha}+1\right)(\alpha, \beta) .
\end{aligned}
$$

This equation involves only $p_{\mathbf{j}}$ with $\mathbf{j} \geq \mathbf{M}$. Define $\operatorname{deg}(\mathbf{j})=\sum_{\alpha \in R^{+}} \mathbf{j}_{\alpha}$ and $|\mathbf{j}|=$ $|\operatorname{deg}(\mathbf{j})|$. Then note that even though some of the $p_{\mathbf{j}}$ are differentiated, none of the ones with $|\mathbf{M}-\mathbf{j}|=1$ are. We can use (7.2) to solve for one of the $p_{\mathbf{j}}$ in terms of $p_{\mathbf{j}}$ of greater degree. If we move through the $\bar{\alpha}^{\mathbf{M}}$ with decreasing degree, we can thus show that all $p_{\mathbf{j}}$ are ultimately determined by $p_{\mathbf{N}}$ alone. It is lengthy although not difficult to give a precise algorithm for how one should progress downward through the indices.

Thus we know that $p_{\mathbf{N}}$ determines $D$. 
Proof of Proposition 5.3. We first note that since $L_{\mathfrak{h}}(k)$ is homogeneous of degree -2 , the generator of $\mathbb{S}_{\mathfrak{h}}(r, k)$ must be homogeneous. If $D \in \mathbb{S}_{\mathfrak{h}}(r, k)$ is not homogeneous, then $D L_{\mathfrak{h}}(k)=L_{\mathfrak{h}}(k+r) D$ implies $\epsilon\left(D L_{\mathfrak{h}}(k)\right)=\epsilon\left(L_{\mathfrak{h}}(k+r) D\right)$, and since $L_{\mathfrak{h}}(k)$ is already homogeneous, $\epsilon(D) L_{\mathfrak{h}}(k)=L_{\mathfrak{h}}(k+r) \epsilon(D)$. So $\epsilon(D)$ is in $\mathbb{S}_{\mathfrak{h}}(r, k)$ but is not a multiple of $D$.

Now since the degree of differential operators is additive, $g(-1, k)$ must have the minimal degree. We know from [10, Corollary 3.12] that $\operatorname{deg}(G(-1, k))=$ $n(n+1) / 2$, and it has lowest homogeneous degree 0 [10, Theorem 4.4]. Thus, since $\mathbf{N}=(1, \ldots, 1)$, we can only have $p_{\mathbf{j}} \neq 0$ for $\mathbf{j}$ whith $\operatorname{deg}\left(p_{\mathbf{j}}\right)=\sum \mathbf{j} \geq 0$.

Now we know $D \in D_{\mathfrak{h}}^{W}$, so $w \cdot p_{\mathbf{j}}(t)=p_{w \cdot \mathbf{j}}(t)$ where $W$ acts on the indices $\mathbf{j}$ by permutation. Thus $p_{\mathbf{N}}$ is $W$-invariant.

Let $\alpha \in R^{+}$. By $(7.2)$ with $\mathbf{M}=(1, \ldots, 1)-2 e_{\alpha}$, we see that

$$
0=2 k p_{\mathbf{N}-e_{\alpha}} t_{\alpha}+\sum_{i=1}^{n} 2 k\left(\partial_{i} p_{\mathbf{N}}\right) t_{\alpha} \operatorname{AV}\left(e_{i}\right)\left(\partial_{i} \alpha\right)-4(1-k) p_{\mathbf{N}}-t_{\alpha} p_{\mathbf{N}-e_{\alpha}} 2(k-1)
$$

since $\mathbf{M}_{\alpha}=-1$. Then we see that $t_{\alpha}$ divides $p_{\mathbf{N}}$ unless

$$
0=2 k p_{\mathbf{N}-e_{\alpha}} t_{\alpha}+\sum_{i=1}^{n} 2 k\left(\partial_{i} p_{\mathbf{N}}\right) t_{\alpha} \operatorname{AV}\left(e_{i}\right)\left(\partial_{i} \alpha\right)-t_{\alpha} p_{\mathbf{N}-e_{\alpha}} 2(k-1) .
$$

This requires $p_{\mathbf{N}}$ to be 0 , which makes $g(-1, k)$ zero, contradicting the fact that it is a generator.

Thus for all $\alpha, t_{\alpha}$ divides $p_{\mathbf{N}}$. Then the positive roots give $n(n+1) / 2$ independent divisors of $p_{\mathbf{N}}$ and $\operatorname{so} \operatorname{deg}(g(-1, k)) \geq n(n+1) / 2$. Thus the $p_{\mathbf{N}}$ term of $g(-1, k)$ is a scalar multiple of the $p_{\mathbf{N}}$ term of $\epsilon(G(-1, k))$. So the operators are scalar multiples of each other and thus $\epsilon(G(-1, k))$ is a generator.

Proof of Proposition 5.1. By Lemma 7.2, $D$ is determined by $p_{\mathbf{N}}(D)$. Given $D \in$ $\mathbb{S}_{\mathfrak{h}}(-1, k)$ and $S \in \mathbb{S}_{\mathfrak{h}}(0, k)$, the lead coefficients are multiplicative,

$$
p_{\mathbf{N}}(S D)=p_{\mathbf{N}}(S) p_{\mathbf{N}}(D) .
$$

Hence Lemma 7.2 reduces the proof to showing that $p_{\mathbf{N}}\left(\mathbb{S}_{\mathfrak{h}}(-1, k)\right)$ is a rank-one $p_{\mathbf{N}}\left(\mathbb{S}_{\mathfrak{h}}(0, k)\right)$-module.

By [7, Theorem 1.7] we know that

$$
\mathbb{C}\left[t_{1}, \ldots, t_{n}\right]^{W} \subset p_{\mathbf{N}}\left(\mathbb{S}_{\mathfrak{h}}(0, k)\right) .
$$

By Proposition 5.3, we know that $\prod_{\alpha \in R^{+}} t_{\alpha}$ divides $p_{\mathbf{N}}(D)$ for all $D \in \mathbb{S}_{\mathfrak{h}}(-1, k)$. Now since $D$ is $W$-invariant and $\mathbf{N}$ is fixed by $W$, we know that the lead term

$$
\left(\prod_{\alpha \in R^{+}} \alpha\right) \partial\left(p_{\mathbf{N}}\right)
$$


is $W$-invariant. Since $w$ acts on $\prod_{\alpha \in R^{+}}$by $\operatorname{sgn}(w)$, this means $w$ acts on $p_{\mathbf{N}}$ by $\operatorname{sgn}(w)$ and is thus equal to

$$
q(t)\left(\prod_{\alpha \in R^{+}} \alpha\right)
$$

where $q(t) \in \mathbb{C}\left[t_{1}, \ldots, t_{n}\right]^{W} \subset p_{\mathbf{N}}\left(\mathbb{S}_{\mathfrak{h}}(0, k)\right)$. Since $p_{\mathbf{N}}(\epsilon(G(-1, k)))=c\left(\prod_{\alpha \in R^{+}} \alpha\right)$, we know $\prod_{\alpha \in R^{+}} \alpha \in p_{\mathbf{N}}\left(\mathbb{S}_{\mathfrak{h}}(-1, k)\right)$. Thus

$$
p_{\mathbf{N}}\left(\mathbb{S}_{\mathfrak{h}}(-1, k)\right)=\mathbb{C}\left[t_{1}, \ldots, t_{n}\right]^{W}\left(\prod_{\alpha \in R^{+}} \alpha\right)
$$

is clearly a rank-one $p_{\mathbf{N}}\left(\mathbb{S}_{\mathfrak{h}}(0, k)\right)$-module.

\section{Acknowledgements}

I would like to thank my advisor, Victor Ginzburg, as my primary mentor in this research. I would also like to thank Eric Opdam, Madhav Nori, Alexander Beilinson, Nero Budur, Mircea Mustaţă, and András Lörincz for their comments, advice, and correspondence.

\section{References}

[1] I. N. Bernšteřn, Modules over a ring of differential operators. An investigation of the fundamental solutions of equations with constant coefficients, Funkcional. Anal. i Priložen. 5 (1971), no. 2, 1-16 (in Russian). Zbl 0233.47031 MR 0290097

[2] M. Finkelberg and V. Ginzburg, Cherednik algebras for algebraic curves, in Representation theory of algebraic groups and quantum groups, Progr. Math. 284, Birkhäuser/Springer, New York, 2010, 121-153. Zbl 1242.14005 MR 2722961

[3] On mirabolic $\mathcal{D}$-modules, Int. Math. Res. Notices 2010, no. 15, 2947-2986. Zbl 1217.14013 MR 2673716

[4] W. L. Gan and V. Ginzburg, Almost-commuting variety, D-modules, and Cherednik algebras, Int. Math. Res. Papers 2006, 26439, 1-54. Zbl 1158.14006 MR 2210660

[5] V. Ginzburg, I. Gordon, and J. T. Stafford, Differential operators and Cherednik algebras, Selecta Math. (N.S.) 14 (2009), 629-666. Zbl 1193.14007 MR 2511194

[6] M. Granger, Bernstein-Sato polynomials and functional equations, in Algebraic approach to differential equations, World Sci., Hackensack, NJ, 2010, 225-291. Zbl 1231.32007 MR 2766095

[7] G. J. Heckman, A remark on the Dunkl differential-difference operators, in Harmonic analysis on reductive groups (Brunswick, ME, 1989), Progr. Math. 101, Birkhäuser Boston, Boston, MA, 1991, 181-191. Zbl 0749.33005 MR 1168482

[8] J. Igusa, An introduction to the theory of local zeta functions, AMS/IP Stud. Adv. Math. 14, Amer. Math. Soc., Providence, RI, 2000. Zbl 0959.11047 MR 1743467

[9] M. Kashiwara, $B$-functions and holonomic systems. Rationality of roots of $B$-functions, Invent. Math. 38 (1976/77), 33-53. Zbl 0354.35082 MR 0430304

[10] E. M. Opdam, Root systems and hypergeometric functions. III, Compos. Math. 67 (1988), 21-49. Zbl 0669.33007 MR 0949270 
[11] _ Some applications of hypergeometric shift operators, Invent. Math. 98 (1989), 1-18. Zbl 0696.33006 MR 1010152

[12] M. Sato, Theory of prehomogeneous vector spaces (algebraic part) - the English translation of Sato's lecture from Shintani's note, Nagoya Math. J. 120 (1990), 1-34. Zbl 0715.22014 MR 1086566

[13] M. Sato and T. Shintani, On zeta functions associated with prehomogeneous vector spaces, Ann. of Math. (2) 100 (1974), 131-170. Zbl 0309.10014 MR 0344230

[14] T. Yano and J. Sekiguchi, The microlocal structure of weighted homogeneous polynomials associated with Coxeter systems. I, Tokyo J. Math. 2 (1979), 193-219. Zbl 0449.58025 MR $0560265 f$ 